www.jmscr.igmpublication.org

Impact Factor (SJIF): 6.379

Index Copernicus Value: 79.54

ISSN (e)-2347-176x ISSN (p) 2455-0450

crossrefDOI: https://dx.doi.org/10.18535/jmscr/v6i9.188

Journal Of Medical Science And Clinical Research

IGM Publication

An Official Publication of IGM Publication

\title{
Clinical Study on the Efficacy of Bhavitha Choorna of Fruit Rind of Vibheetaki (Terminalia Bellerica (Gaertn.) Roxb.) in Hyperuricemia
}

\author{
Authors \\ Midhun.M' ${ }^{1}$, Dr P Y Ansary ${ }^{2}$ \\ ${ }^{1}$ PG Scholar, Dept. of Dravyaguna Vijnanam, Govt. Ayurveda College, Tripunithura \\ ${ }^{2}$ Professor \& HOD, Dept. of Dravyaguna Vijnanam, Govt. Ayurveda College, Tripunithura \\ Corresponding Author
}

Dr Midhun.M

PG scholar, Department of Dravyagunavijnana, Government Ayurveda College, Tripunithura, Kerala, India Email: midhun.m.nair@gmail.com,Ph. No: 9496348883

\begin{abstract}
Hyperuricemia is clinical condition in which Serum uric acid level exceeds $7 \mathrm{mg} / \mathrm{dL} \mathrm{L}^{1}$. It is characterised by pain, tenderness and swelling of affected joints. Uric acid metabolism follows different pathways. Uric acid is the end product of purine metabolism. Purins are derived partly from the diets (organ meat, meat extracts, yeast, legumes, fish, beer, mushrooms etc.) and partly from endogenous metabolism. In the liver nucleic acids and purine nucleotides are degraded to form the purine bases xanthine and hypoxanthine ${ }^{2}$. These are oxidised to form uric acid by enzyme xanthine oxidase. The urates circulate in the plasma to be excreted mainly by the kidneys. Increased production of uric acid, reduced urinary elimination or a combination of both these processes lead to hyperuricemia. In the Ayurvedic concept, Rasa, Rakta dhatu dushti and srotodushti due to improper digestion is responsible for the condition. Vibheetaki is a drug described extensively in Ayurvedic classics can correct these conditions at dathu and sroto level. In this study an attempt is made to analyse the efficacy of fruit rind of Vibheetaki in Hyperuricemia and associated conditions

Keywords: Terminalia bellerica (Gaertn.)Roxb, Bhavitha Vibheetaki, Hyperuricemia.
\end{abstract}

\section{Introduction}

Vibheetaki (Terminalia bellerica (Gaertn.)Roxb) is a drug described extensively in Brhattrayis, Lagutrayis as well as in Nighantus. According to Acharya Caraka ${ }^{3}$, Vibheetaki is beneficial to cure pathological conditions related to rasa, rakta, mamsa, medo dathus. Acharya Susrutha described the doshic action of Vibheetaki as kaphapitha samana. Acharya Susruta ${ }^{4}$ indicated the fruit kalkam as a remedy in pathological conditions related to mootra (urine) and asmari (calculus).
Hence the fruits of Vibheetaki may be beneficial in correcting the metabolism of purins, the process of elimination of uric acid through urine

\section{Materials and Methods}

\section{Methods of Clinical Study}

\section{Selection of patients}

Patients were selected as per the inclusion and exclusion criteria from Out Patient Department of Dravyaguna vijnana, Government Ayurveda College, Thripunithura. 
a) Inclusion criteria

1. Subjects having blood uric acid level above $7 \mathrm{mg} / \mathrm{dl}$.

2. Age group between 18-60 years.

3. Patient willing to give written consent.

b) Exclusion criteria

1. Pregnant and lactating women.

2. Patients of age below 18 years.

3. Mentally retarded, blind, and deaf patients.

\section{Sampling method}

a) Sample frame

i. Sample size- 30

ii. Sampling technique- purposive sampling

iii. Study design - Interventional pre \& post-test without control group

iv. Selection of patients- As per inclusion \& exclusion criteria

v. Period of study- 18 month

b) Procedure

30 patients from the outpatient department

of Government Ayurveda College Hospital, Thripunithura fulfilling the inclusion and exclusion criteria will be selected. Lab investigations will be done as per the assessment criteria. Objective evaluation will be done before treatment and on every $30^{\text {th }}$ day up to 3 months.

\section{Methods of Assessment Criteria}

Both pre and post assessment of the patient was done on the basis of clinical symptoms and necessary investigations.

\section{Objective criteria}

Laboratory investigation

Blood uric acid level

The above mentioned investigation was carried out before and after treatment. Changes in the values were recorded in the case proforma in each visit, every 30 days for 3 months for the assessment.

\section{Subjective criteria}

Assessment of therapy was done based on the relief observed in the signs and symptoms with the help of the following scoring pattern which was extracted from the Hutchinson's Clinical Methods.

\section{Pain}

Grading of pain was given on the basis of Visual Analogue Scale (VAS) of $100 \mathrm{~mm}$.

0 $100 \mathrm{~mm}$

\begin{tabular}{ll} 
No Pain & \multicolumn{1}{c}{ Severe Pain } \\
Grade 0 - & No pain, 0-4 $\mathrm{mm}$ \\
Grade $1-$ & Mild pain, 5-44 $\mathrm{mm}$ \\
Grade 2 - & Moderate pain, 45-74 mm \\
Grade 3 - & Severe pain, 75-100 mm
\end{tabular}

\section{Tenderness}

Grade 0 - No tenderness

Grade 1 - The patient says the joint is tender

Grade 2 - Pain and wincing of face on pressure

Grade 3 - Pain and withdrawal of affected part on pressure

Grade 4- The patient will not allow the joint to be touched

\section{Swelling}

Grade 0 - No swelling

Grade 1 - Slightly obvious

Grade 2 - Covers well bony prominence

Grade 3 - Much elevated so that joint is gross deformed

\section{Methods of data Collection}

The data was collected by interrogation, observation and laboratory investigations and recorded in a prepared Clinical case study proforma. The following data were used for analysis as per protocol from assessment of patients.

a) Demographic data

b) Data relating to personal history

c) Data relating to clinical picture

d) Data relating to intervention

The data collected were entered in appropriate tables for easy statistical evaluation. 


\section{Variables}

Results analysed based on Outcome variables and Study variables.

Outcome variables

1. Blood uric acid level.

2. Pain

3. Tenderness

4. Swelling

Study variables include Age, Sex, Religion, Socioeconomic status, Educational status, Occupation, Marital status, Health status, and Nutritional status.

\section{Data Analysis}

The data obtained was analysed and the suitable statistical method was adopted to ascertain the clinical and statistical significance of the work. Paired ' $t$ ' test was applied for the objective parameter. Graphs were plotted based on the findings to make the interpretations better.

\section{Ethical Considerations}

The study was begun after getting the approval from the Institutional Ethical Committee. An informed consent was obtained from all patients before trial

\section{Method of Preparation of Medicine}

Bhavitha choorna of fruit rind of Vibheetaki will be prepared according to the reference in Bhaishajyaratnavali ${ }^{5}$. Fruit rind of Vibheetaki collected from the market will be washed thoroughly and dried. Then it is finely powdered to prepare the choorna. Sufficient quantity of kasaya of the fruit rind of Vibheetaki is prepared in the following ratio of boiling $48 \mathrm{~g}$ of thoroughly washed and crushed fruit rinds with 8 times of water. Then it will be reduced to $1 / 8^{\text {th }}$. Bhavana will be done by fully soaking the fine powder in the kasaya, and drying in shade to avoid the loss of essential phytochemicals. After attaining proper dryness, drug will be made into fine powder of mesh size-120. This process will be repeated three times for the preparation of Bhavitha choorna of fruit rind of Vibheetaki. The fine powder of Bhavitha choorna will be filled in the capsule of $500 \mathrm{mg}$ and will be kept in air tight bottles

Fig 1: Preparation of Medicine

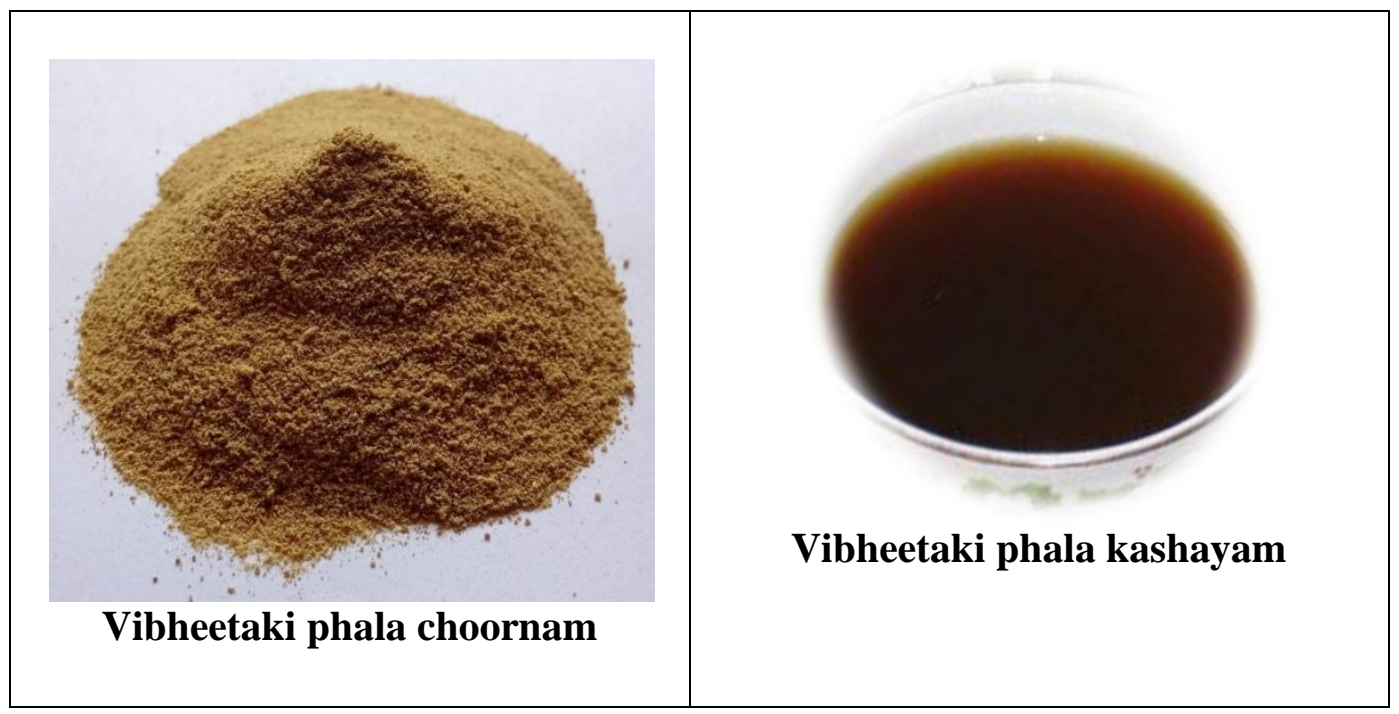




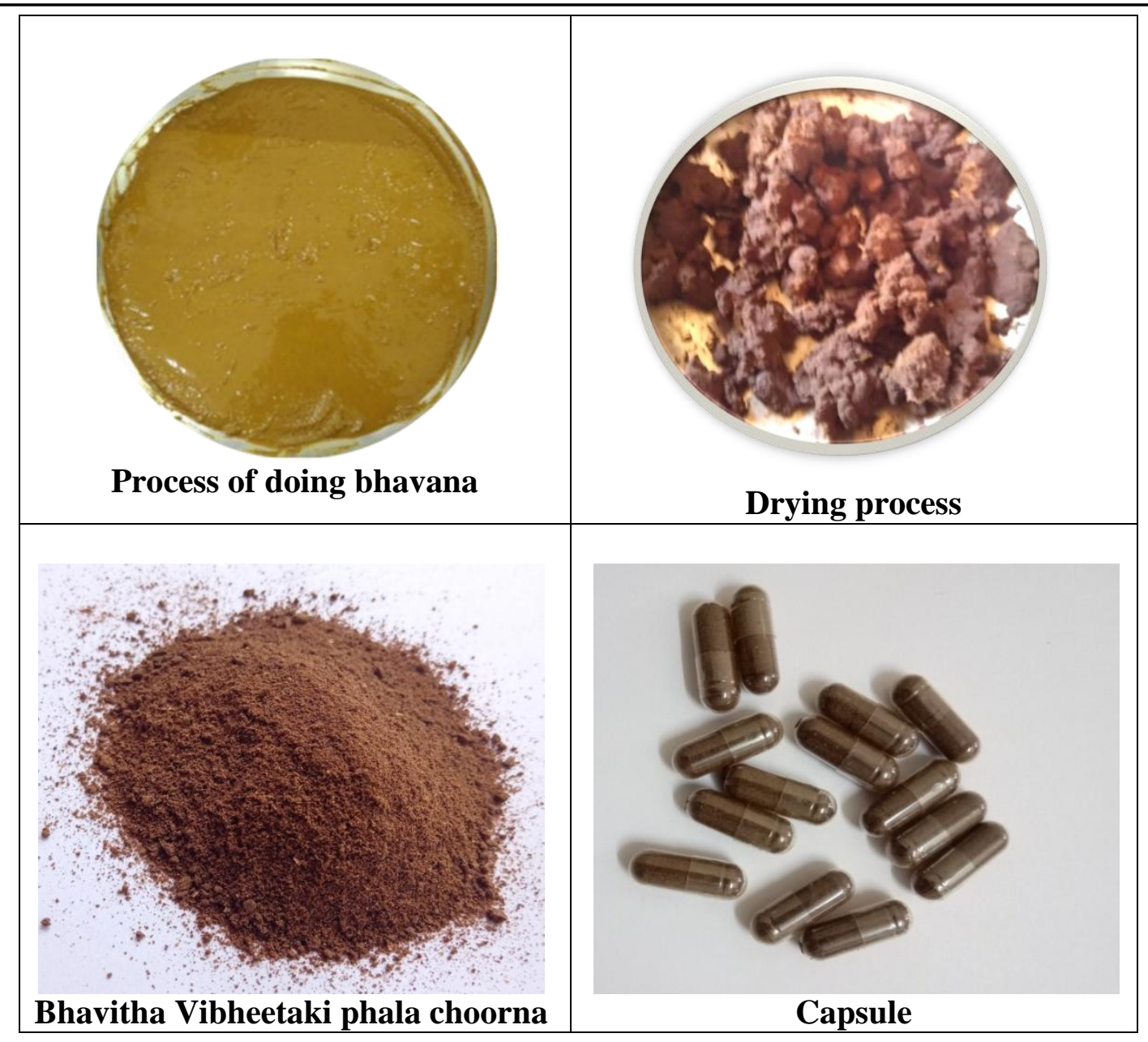

\section{Procedure of Study}

Table 1: Procedure of Study

\begin{tabular}{|l|l|}
\hline Form of medication: & Capsule \\
\hline Dose: & $\begin{array}{l}2 \text { capsule (each of 500mg) } \\
\text { twice daily (8AM \& 8PM). }\end{array}$ \\
\hline Anupana: & Boiled and cooled water \\
\hline Time of administration: & After food \\
\hline Duration: & 3 months \\
\hline Follow up: & $\begin{array}{l}\text { After } 3 \text { months without } \\
\text { medication }\end{array}$ \\
\hline Period of study: & 18 months \\
\hline
\end{tabular}

\section{Results}

Effectiveness of treatment on symptoms of hyperuricemia

In this section, data on symptoms of urinary tract infection were collected before the intervention, $0^{\text {th }}$ day (pre-test), every $30^{\text {th }}$ day up to 3 months and another 3 months follow up. The effectiveness of treatment is statistically assessed using paired $t$ test. For continuous variables, mean and Standard Deviations were calculated and for categorical variation, frequency and percentage were calculated as summary measures. The calculated $\mathrm{p}$ value less than 0.05 is considered to be statistically significant. The details are given in the following tables.

\section{Objective criteria}

The data on the mean, standard deviation of the objective criteria serum uric acid level is tabulated. The percentage change and the significance are statistically analysed.

Table 2: Change in Serum uric acid level after Treatment

\begin{tabular}{|l|c|c|c|}
\hline Serum uric acid & Mean & SD & p-value \\
\hline Before Treatment & 7.657 & 1.153 & \\
\hline After Treatment & 6.410 & 1.073 & $<.0001$ \\
\hline Follow up & 6.583 & 0.8518 & 0.04 \\
\hline
\end{tabular}

Here the p-value after the treatment is less than .0001 that shows the change in the serum uric acid is extremely significant and the p-value after follow up is 0.04 that means the change in serum uric acid level considered as significant. The diagrammatic representation of serum uric acid level before and after treatment is shown in fig. 2 . 
Figure 2: Change in Serum uric acid after Treatment

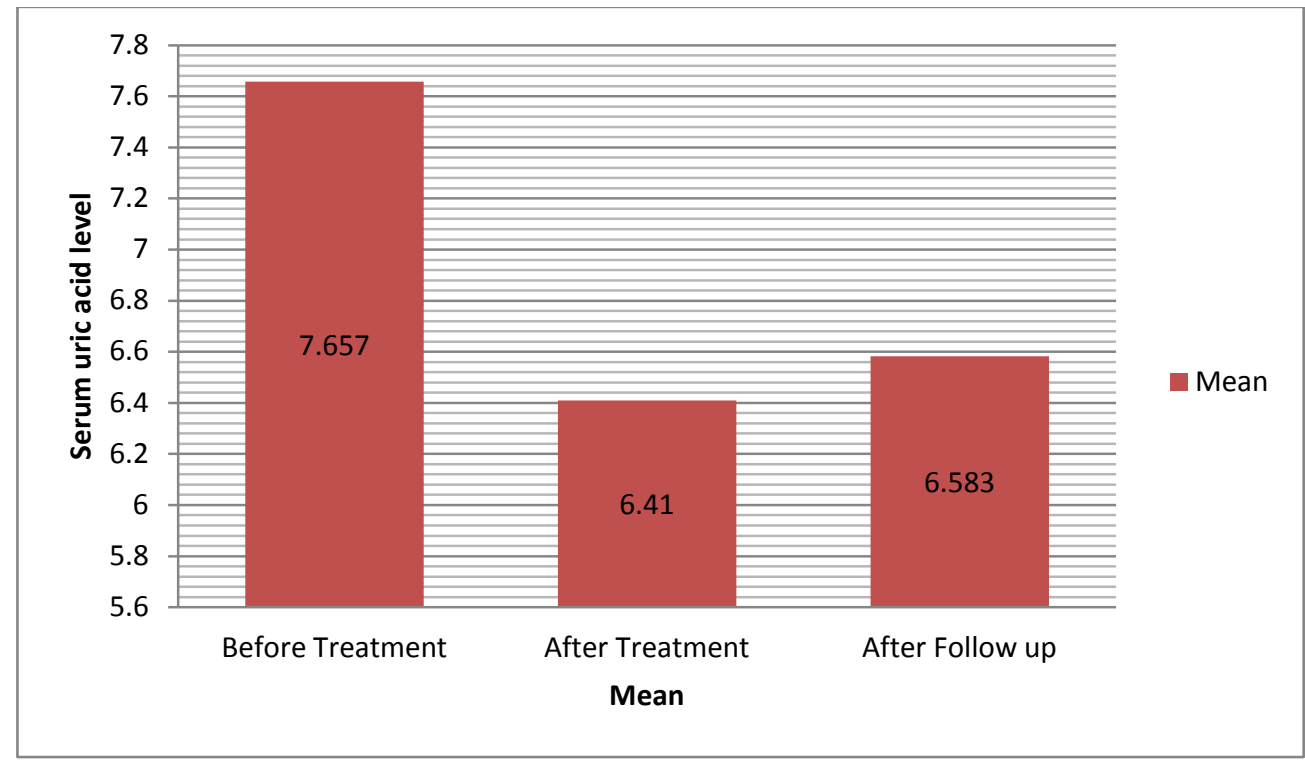

\section{Subjective criteria}

The data on the subjective parameters such as pain, tenderness, swelling were tabulated. The transition of assessment grades and the frequency were calculated and the significance was assessed statistically.

Table 3: Change in Pain after Treatment

\begin{tabular}{|l|c|c|c|}
\hline Pain & Before Treatment & After Treatment & p - value \\
\hline Grade 0 & $0(0.0 \%)$ & $23(76.67 \%)$ & \\
\cline { 1 - 3 } Grade 1 & $7(23.33 \%)$ & $5(16.67 \%)$ & \multirow{2}{*}{$<0.0001$} \\
\hline Grade 2 & $20(66.67 \%)$ & $2(6.67 \%)$ & \\
\cline { 1 - 3 } Grade 3 & $3(10 \%)$ & $0(0.0 \%)$ & \\
\hline Grade 4 & $0(0.0 \%)$ & $0(0.0 \%)$ & \\
\hline
\end{tabular}

Here the p-value is less than the significance level 0.0001 ; hence the change in pain after treatment is extremely significant. The table shows that $76.67 \%$ cases are reduced to grade $0,16.67 \%$ cases are reduced to grade 1 , and $6.67 \%$ of cases are reduced to grade 2 after treatment. The diagrammatic representation of change in the pain is shown in figure 3.

Figure 3: Change in Pain after Treatment

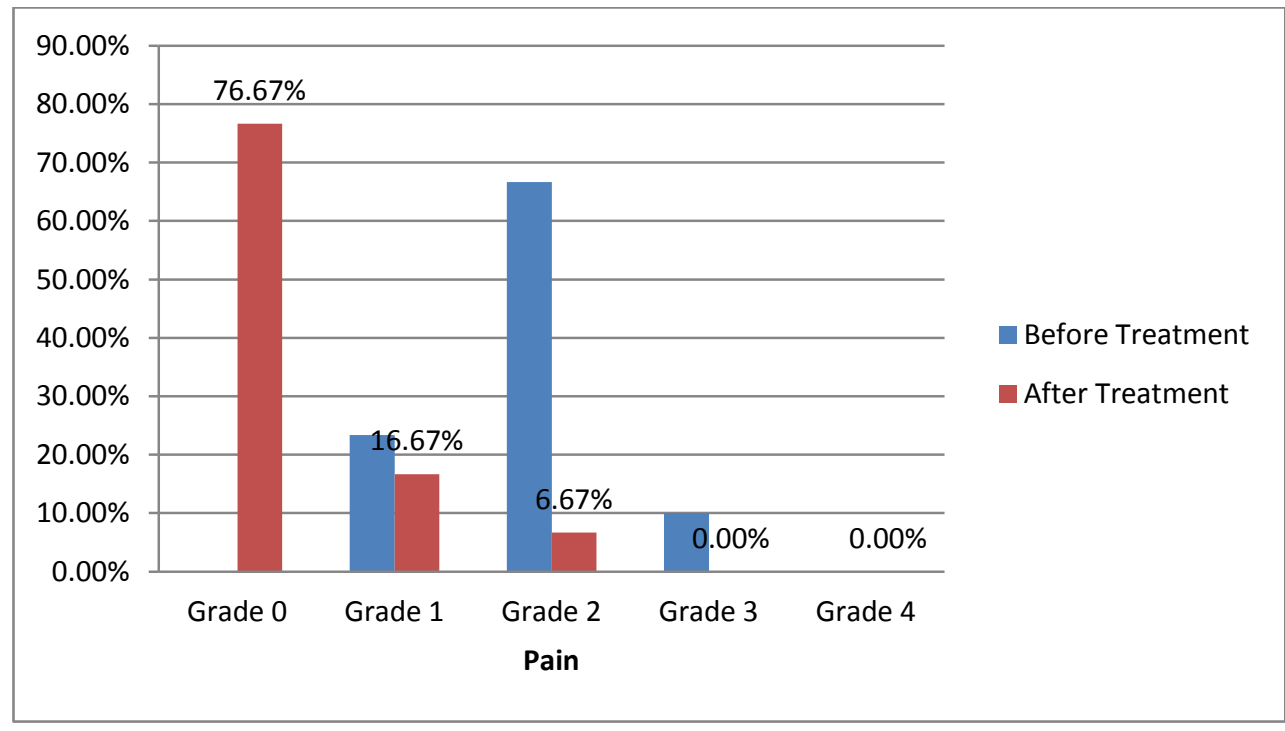


Table 4: Change in Tenderness after Treatment

\begin{tabular}{|l|c|c|c|}
\hline Tenderness & Before Treatment & After Treatment & \multirow{2}{*}{ p-value } \\
\hline Grade 0 & $8(26.67 \%)$ & $22(73.33 \%)$ & \\
\cline { 1 - 3 } Grade 1 & $11(36.67 \%)$ & $7(23.33 \%)$ & \\
\cline { 1 - 2 } Grade 2 & $8(26.67 \%)$ & $1(3.33 \%)$ & \multirow{2}{*}{$<0.0001$} \\
\hline Grade 3 & $2(6.67 \%)$ & $0(0.0 \%)$ & \\
\hline Grade 4 & $1(3.33 \%)$ & $0(0.0 \%)$ & \\
\hline
\end{tabular}

Here the p-value is less than the significance level 0.0001; hence the change in tenderness after treatment is extremely significant. The table shows that $73.33 \%$ cases are reduced to grade 0 , Figure 4: Change in Tenderness after Treatment

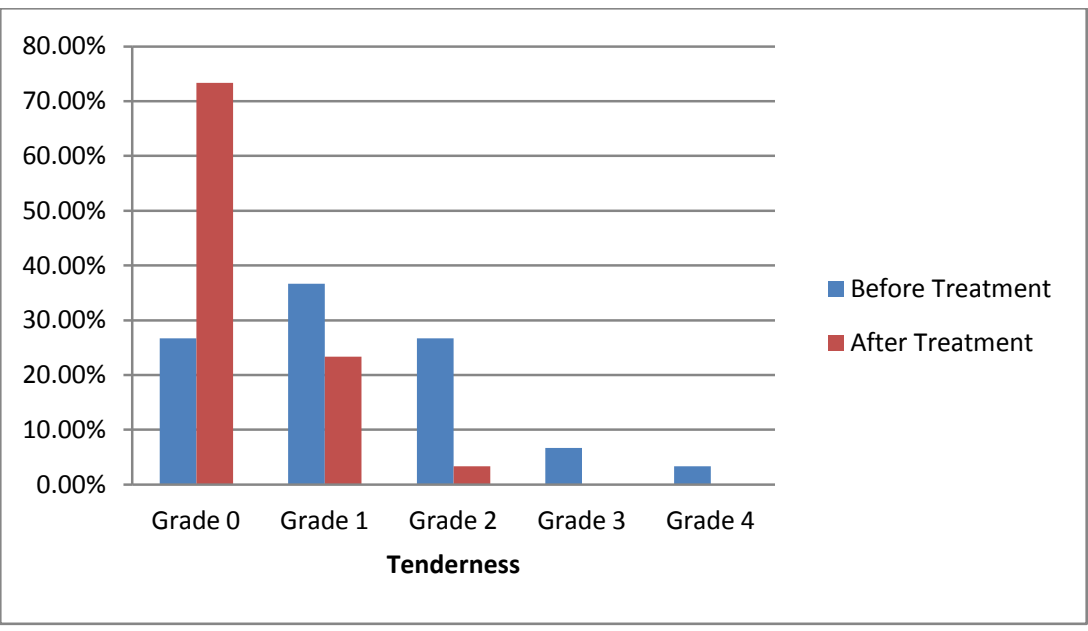

Table 5: Change in Swelling after Treatment

\begin{tabular}{|l|c|c|c|}
\hline Swelling & Before Treatment & After Treatment & p - value \\
\hline Grade 0 & $6(20 \%)$ & $26(86.67 \%)$ & \\
\cline { 1 - 3 } Grade 1 & $21(70 \%)$ & $4(13.33 \%)$ & \multirow{2}{*}{$<0.0001$} \\
\cline { 1 - 3 } Grade 2 & $3(10 \%)$ & $0(0.0 \%)$ & \\
\cline { 1 - 3 } Grade 3 & $0(6.67 \%)$ & $0(0.0 \%)$ & \\
\hline Grade 4 & $0(3.33 \%)$ & $0(0.0 \%)$ & \\
\hline
\end{tabular}

Here the p-value is less than the significance level 0.0001; hence the change in swelling after treatment is extremely significant. The table shows that $86.67 \%$ cases are reduced to grade 0 ,
$23.33 \%$ cases are reduced to grade 1 , and $3.33 \%$ of cases are reduced to grade 2 after treatment. The diagrammatic representation of change in the tenderness is shown in figure 4 .

Figure 5: Change in Swelling after Treatment

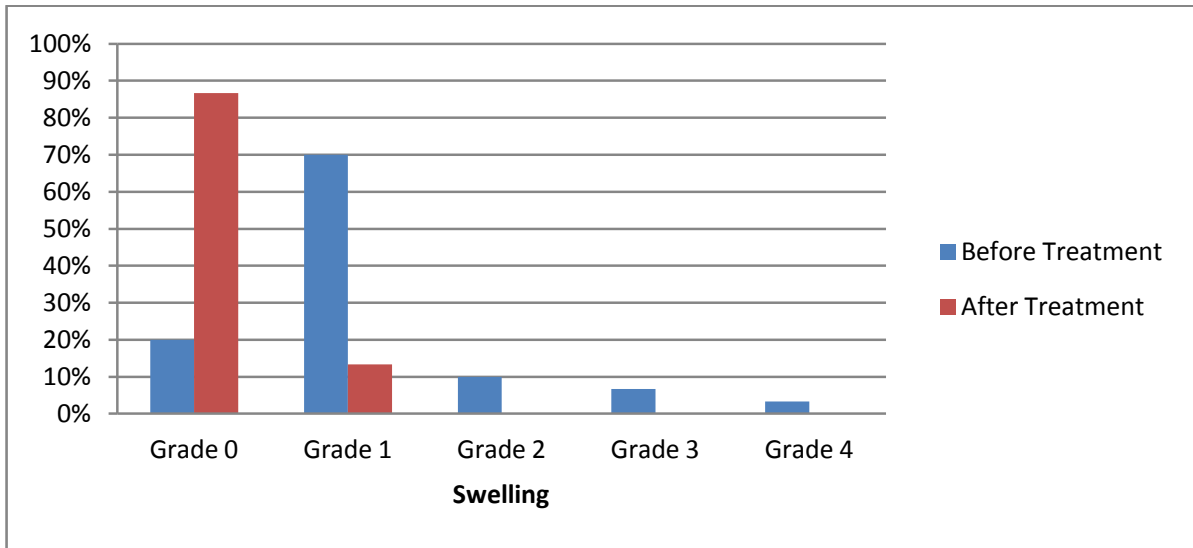




\section{Discussion}

In the lights of Ayurveda various aspects of Hyperuricemic conditions associated with clinical symptoms from Ayurvedic and modern perspective have been recognized, explored and discussed in this study. This is an attempt made to critically discuss and understand the whole clinical study regarding "Clinical study on the efficacy of bhavitha choorna of Vibheetaki (Terminalia bellerica (Gaertn.) Roxb) phala in Hyperuricemia.

\section{Effectiveness of the trial on Serum uric acid}

Effectiveness of treatment in 3 months is calculated at the end using Paired t test. Firstly compare Serum uric acid levels before and after treatment. Mean of the two values are 7.657 and 6.41 respectively, and the $\mathrm{p}$ value obtained is less than 0.0001 , that means the change in the serum uric acid level before and after treatment is extremely significant. Secondly compared the serum uric acid levels after treatment and a follow up period for 3 months, here the mean of two groups are 6.41 and 6.583 respectively and the $p$ value obtained is 0.04 which is less than the significance level 0.05 . That means the change in serum uric acid level after treatment and during follow up is significant and it shows that the effect of the treatment is maintained during the follow up period.

\section{Probable mode of action}

The doshic action of Vibheetaki is kapha-pitta samana, since hyperuricemia is a condition in which is initiated with the production of Ama and is mainly affected rasa and raktadhatus, the kapha-pitta samana action of the drugs helps to cure the condition. According to Acharya Vagbhata, Vibheetaki possess the same properties of Hareetaki in less amount and has got actions on agni as deepana and on ama as pacana. Laghu and ruksa gunas of the drug helps in amapacana and kaphasamana. According to Acharya Susruta the fruit kalkam of Vibheetaki is used as a remedy in the pathological conditions related to mootra (urine) and in asmari (calculus). Since Hyperuricemia is also caused by the inadequate excretion of Uric acid through urine, the action of the drug in mootrados has may helpful. Hyperuricemia is progressed with the more and more deposition of urate crystals in the synovium of joints, with the asmarighna property the drug may act to dissolve the urate crystals and facilitates its excretion through urine. Moreover a reported randomized, double blind, and placebocontrolled human clinical trial has recently confirmed the dose-dependent action of Terminalia bellerica extract in decreasing blood levels of uric acid ${ }^{6}$.

\section{Conclusion}

The clinical study showed statistically significant results for the objective and subjective criteria. The efficacy of the treatment was also maintained during the follow up period as well. In the present situations, increasing prevalence of obesity, diuretic use, adverse lifestyle and dietary habits, and an ageing population may all be contributing to recent increases in the prevalence of hyperuricemia, and it leads to many systemic diseases like gouty arthritis which is a very painful condition. So an early control of the disease is necessary. Allopathic medicines presently used for the management of hyperuricemia are Febuxostat, Allopurinol etc, which are reported with serious side effects ranging from breathing difficulties to heart attacks and strokes, and also reoccurrence of the disease are reported with the withdrawal of medication. It demands the need of an effective alternative medicine which is free of side effects. Here comes the importance of the drug Vibheetaki as it fulfils all the above needs and effectively controls the Serum uric acid level and can manage the symptoms associated with it in a very natural way.

\section{Acknowledgement}

I express my immense respect and gratitude towards Dr. P.Y. Ansary, my guide and the Head of the department of Dravyaguna vijnana, Govt. Ayurveda College, Thripunithura, for his immense guidance and motivation throughout the work. 


\section{References}

1. Krishnadas Book of Medicine by Dr. K.V. Krishnadas, $5^{\text {th }}$ Edition, in Metabilic arthropathies, Page: 729.

2. Cecil-Loeb Text Book of Medicine by Beeson and McDermott, in Disorders of Purine Metabolism- Lloyd.H Smith.jr, Page: 1688

3. Caraka Samhitha Sutra sthana, English translation, with Cakrapani Datta's Ayurveda Dipika Commentary, by R.K.Sharma and Bhagwan Dash, Chapter 27, Page:516

4. Susrutha Samhitha Sutra sthana, English translation, by Prof.Sreekanda Murthy, Chapter 46, Page:402

5. Bhaishajyaratnavali, English translation, Vol-1, Chapter-4, Sloka:119, Page:90

6. Life Extension Magazine- Prevent Gout Attacks by Lowering Uric Acid Levels, and Rani.U, Kishan.P, Chandrasekharan.N. A randomized, double blind, placebo controlled, parallel group study to evaluate the effect of Terminalia chebula, Terminalia bellerica and Febuxostat in patients with hyper urecemia. 